\title{
Advanced Oxidation Processes for Water Treatment
}

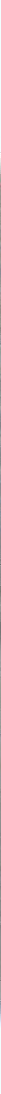

FUNDAMENTALS AND APPLICATIONS

Edited by Mihaela I. Stefan 


\section{Advanced Oxidation Processes for Water Treatment}





\section{Advanced Oxidation Processes for Water Treatment}

Fundamentals and Applications

Edited by

Mihaela I. Stefan

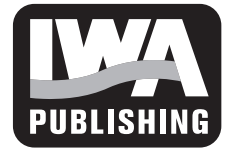


Published by

(C) 2018 IWA Publishing

\author{
IWA Publishing \\ Alliance House \\ 12 Caxton Street \\ London SW1H 0QS, UK \\ Telephone: $+44(0) 2076545500$ \\ Fax: +44 (0)20 76545555 \\ Email: publications@iwap.co.uk \\ Web: www.iwapublishing.com
}

Apart from any fair dealing for the purposes of research or private study, or criticism or review, as permitted under the UK Copyright, Designs and Patents Act (1998), no part of this publication may be reproduced, stored or transmitted in any form or by any means, without the prior permission in writing of the publisher, or, in the case of photographic reproduction, in accordance with the terms of licenses issued by the Copyright Licensing Agency in the UK, or in accordance with the terms of licenses issued by the appropriate reproduction rights organization outside the UK. Enquiries concerning reproduction outside the terms stated here should be sent to IWA Publishing at the address printed above.

The publisher makes no representation, express or implied, with regard to the accuracy of the information contained in this book and cannot accept any legal responsibility or liability for errors or omissions that may be made.

\section{Disclaimer}

The information provided and the opinions given in this publication are not necessarily those of IWA and should not be acted upon without independent consideration and professional advice. IWA and the Editors and Authors will not accept responsibility for any loss or damage suffered by any person acting or refraining from acting upon any material contained in this publication.

British Library Cataloguing in Publication Data

A CIP catalogue record for this book is available from the British Library

ISBN: 9781780407180 (Paperback)

ISBN: 9781780407197 (eBook)

\section{Cover images:}

TrojanUV system at Orange County Water District, CA, USA. Courtesy of Dr. George Tchobanoglous, UC Davis, CA, USA RO Membrane filtration system at Orange County Water District, CA, USA. Courtesy of OCWD

WEDECO PDO 1000 ozone generator installed at Sung-Nam water treatment plant, South Korea. Courtesy of WEDECO, a Xylem brand

All other images from istockphoto.com 
To all of those who dream big, believe in themselves and work hard to make a difference in the world.

My thoughts go to my parents who taught me the value of perseverance despite humble beginnings and to my family who supported me on this journey.

Mihaela I. Stefan

August 3, 2017 



\section{Contents}

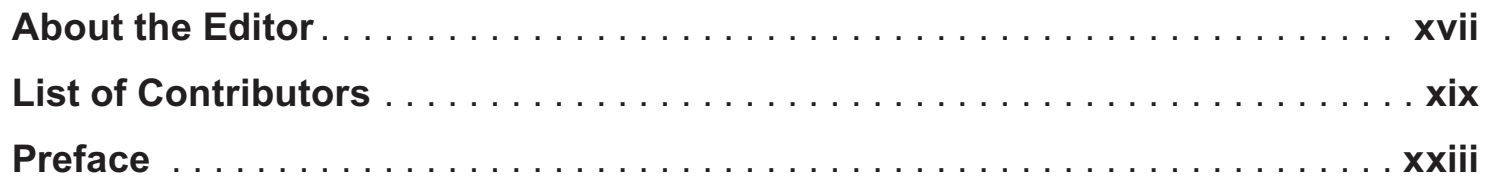

\section{Chapter 1}

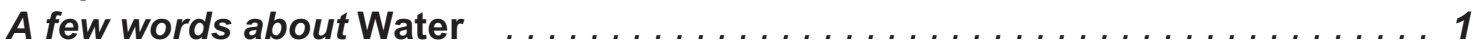

Mihaela I. Stefan

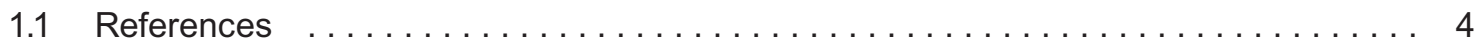

\section{Chapter 2}

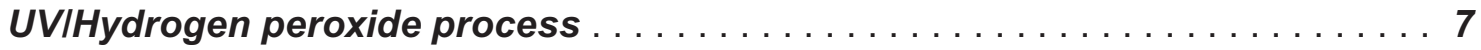

Mihaela I. Stefan

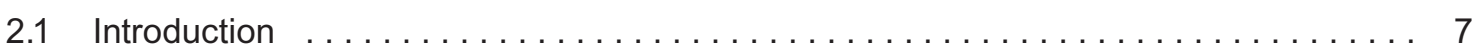

2.2 Electromagnetic Radiation, Photochemistry Laws and Photochemical Parameters . . 8

2.2.1 Electromagnetic radiation $\ldots \ldots \ldots \ldots \ldots \ldots \ldots \ldots \ldots \ldots \ldots$

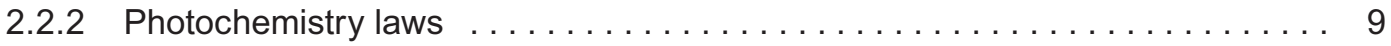

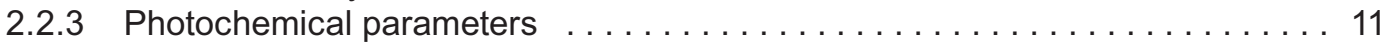

2.3 UV Radiation Sources . . . . . . . . . . . . . . . . . . . . . . . . . . . 15

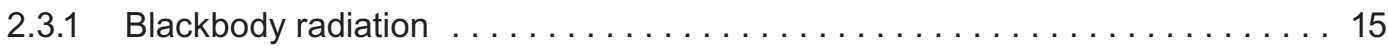

2.3.2 Mercury vapor-based UV light sources for water treatment $\ldots \ldots \ldots \ldots \ldots 16$

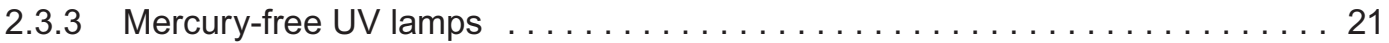

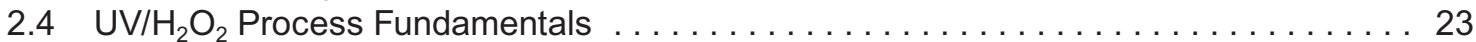

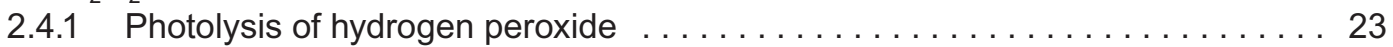

2.4 .2 Hydroxyl radical . . . . . . . . . . . . . . . . . . . . 27

2.4.3 Rate constants of ${ }^{\circ} \mathrm{OH}$ reactions with organic and inorganic compounds ... 32 


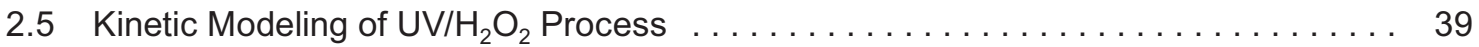

2.5.1 Pseudo-steady-state approximation and dynamic kinetic models . . . . . 40

2.5.2 Computational fluid dynamics models for the $\mathrm{UV} / \mathrm{H}_{2} \mathrm{O}_{2}$ process $\ldots \ldots \ldots .46$

2.6 Water Quality Impact on $\mathrm{UV} / \mathrm{H}_{2} \mathrm{O}_{2}$ Process Performance $\ldots \ldots \ldots \ldots \ldots \ldots$

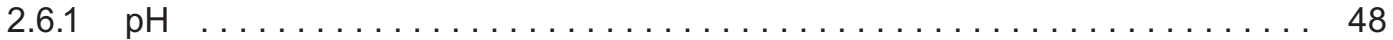

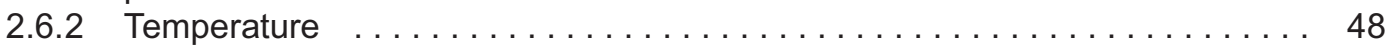

2.6.3 Water matrix composition $\ldots \ldots \ldots \ldots \ldots \ldots \ldots \ldots \ldots \ldots \ldots \ldots \ldots \ldots$

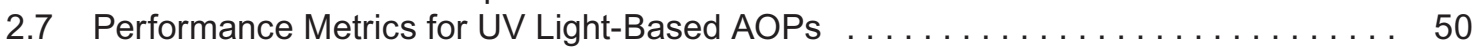

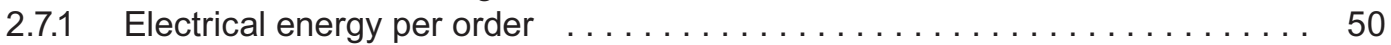

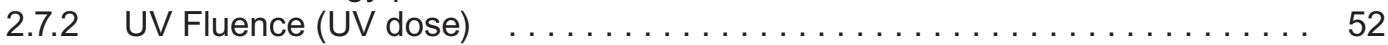

$2.8 \mathrm{UV} / \mathrm{H}_{2} \mathrm{O}_{2}$ AOP Equipment Design and Implementation $\ldots \ldots \ldots \ldots \ldots \ldots \ldots \ldots \ldots$

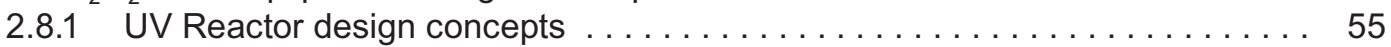

2.8.2 Sizing full-scale UV equipment from bench- and pilot-scale $\ldots \ldots \ldots \ldots$. . 57

2.8.3 Incorporating the UV light-based processes into water treatment trains . . . 59

$2.9 \mathrm{UV} / \mathrm{H}_{2} \mathrm{O}_{2}$ AOP for Micropollutant Treatment in Water $\ldots \ldots \ldots \ldots \ldots \ldots$

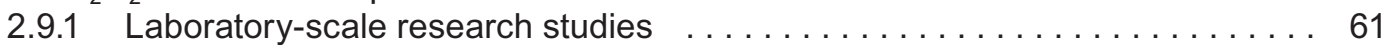

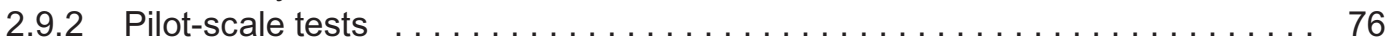

2.9.3 Full-scale $U V / \mathrm{H}_{2} \mathrm{O}_{2}$ AOP installations $\ldots \ldots \ldots \ldots \ldots \ldots \ldots \ldots \ldots \ldots \ldots$

2.9.4 Process economics, sustainability and life-cycle assessment $\ldots \ldots \ldots 8$

2.10 Byproduct Formation and Mitigation Strategies $\ldots \ldots \ldots \ldots \ldots \ldots \ldots$

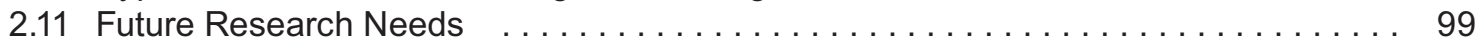

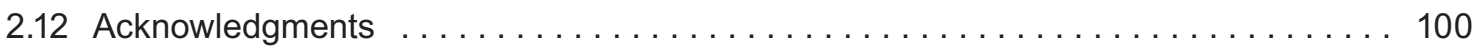

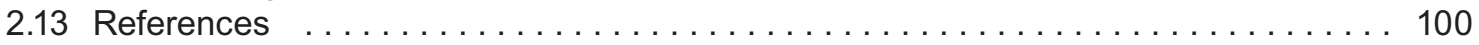

\section{Chapter 3}

Application of ozone in water and wastewater treatment . . . . . . . . . . 123 Daniel Gerrity, Fernando L. Rosario-Ortiz, and Eric C. Wert

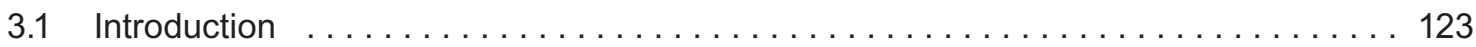

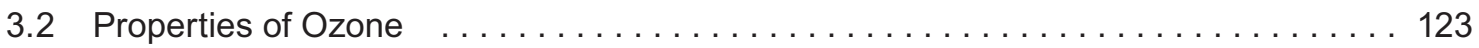

3.3 Decomposition of Ozone in Water . . . . . . . . . . . . . . . . . . . . . . . . . . . . 124

3.4 Ozonation for Contaminant Removal $\ldots \ldots \ldots \ldots \ldots \ldots$

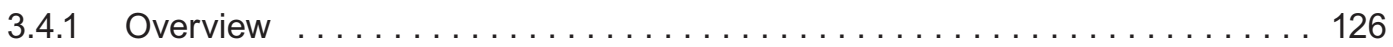

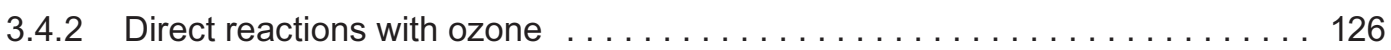

3.4.3 Impact of water quality on process performance $\ldots \ldots \ldots \ldots \ldots \ldots \ldots$

3.4 .4 Summary . . . . . . . . . . . . . . . . . . . . . . . . 138

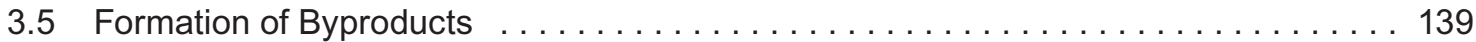

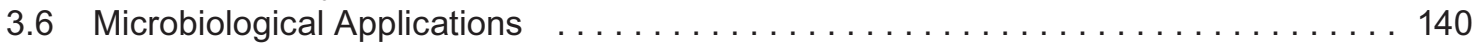

3.6.1 Disinfection in drinking water and wastewater applications $\ldots \ldots \ldots \ldots \ldots$

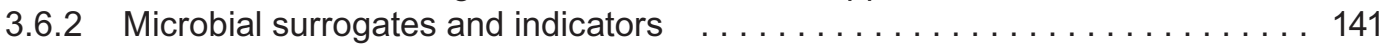

3.6.3 Ozone dosing frameworks for disinfection $\ldots \ldots \ldots \ldots \ldots \ldots \ldots \ldots$

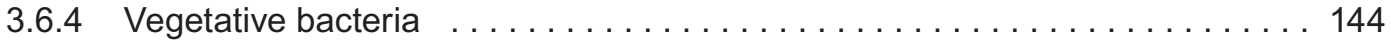

3.6 .5 Viruses . . . . . . . . . . . . . . . . . . . . . . . . 146

3.6.6 Spore-forming microbes $\ldots \ldots \ldots \ldots \ldots \ldots \ldots \ldots \ldots \ldots \ldots \ldots \ldots \ldots$

3.7 Implementation at Full Scale Facilities $\ldots \ldots \ldots \ldots \ldots \ldots \ldots \ldots$

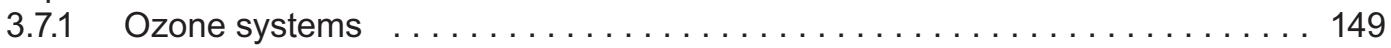




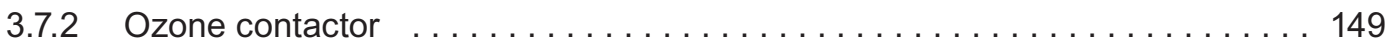

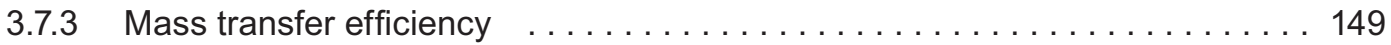

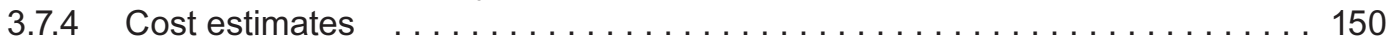

3.7 .5 Process control . . . . . . . . . . . . . . . . . . . . . . . . . . . . 152

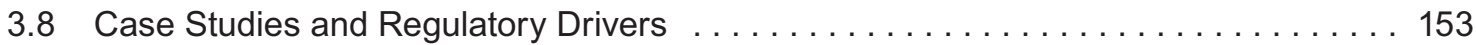

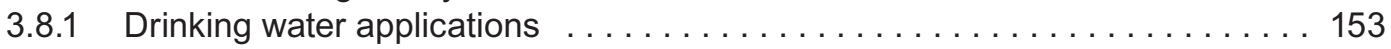

3.8.2 Wastewater and potable reuse applications $\ldots \ldots \ldots \ldots \ldots \ldots \ldots \ldots$

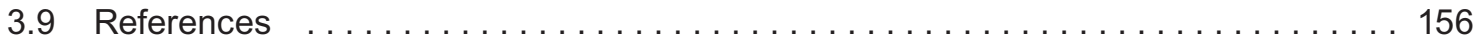

\section{Chapter 4}

Ozone/ $\mathrm{H}_{2} \mathrm{O}_{2}$ and ozone/UV processes

Alexandra Fischbacher, Holger V. Lutze and Torsten C. Schmidt

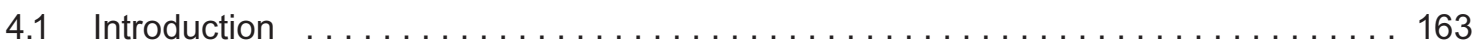

$4.2 \mathrm{O}_{3} / \mathrm{H}_{2} \mathrm{O}_{2}$ (Peroxone) Process Fundamentals . . . . . . . . . . . . . . . . 163

4.2.1 Mechanism of hydroxyl radical generation $\ldots \ldots \ldots \ldots \ldots \ldots \ldots \ldots \ldots$

$4.2 .2 \mathrm{O}_{3}$ and $\cdot \mathrm{OH}$ exposures: the $R_{\mathrm{ct}}$ concept $\ldots \ldots \ldots \ldots \ldots \ldots \ldots \ldots \ldots \ldots \ldots \ldots \ldots \ldots \ldots$

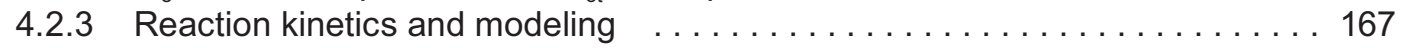

4.2.4 Water quality impact on process performance:

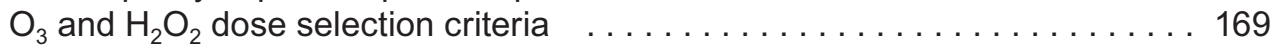

$4.3 \quad \mathrm{O}_{3} / \mathrm{H}_{2} \mathrm{O}_{2}$ AOP for Micropollutant Removal $\ldots \ldots \ldots \ldots \ldots \ldots \ldots \ldots \ldots \ldots \ldots \ldots$

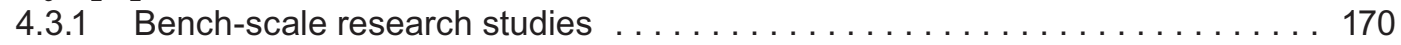

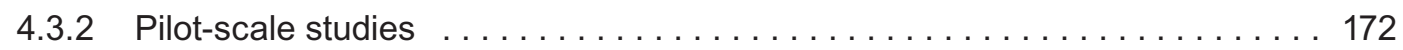

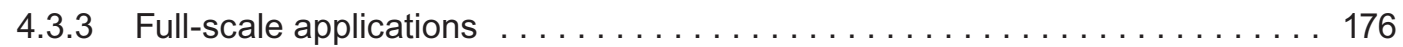

4.3.4 Process economics and limitations $\ldots \ldots \ldots \ldots \ldots \ldots \ldots \ldots \ldots \ldots \ldots \ldots \ldots$

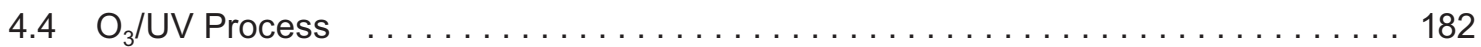

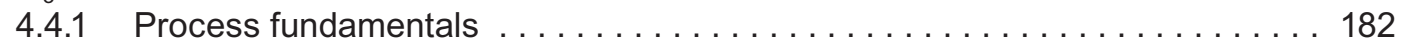

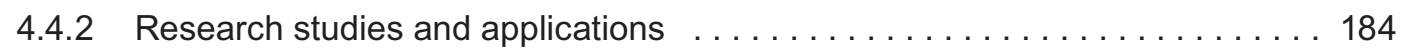

4.5 Byproduct Formation and Mitigation Strategies $\ldots \ldots \ldots \ldots \ldots \ldots \ldots \ldots \ldots \ldots \ldots$

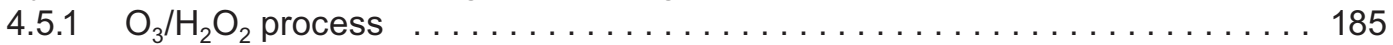

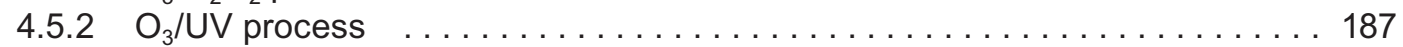

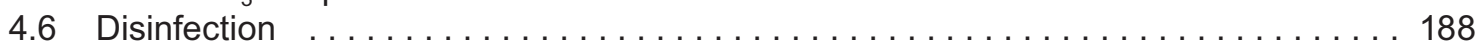

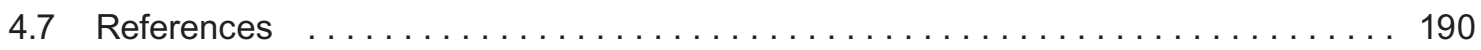

\section{Chapter 5}

Vacuum UV radiation-driven processes . . . . . . . . . . . . . . 195

Tünde Alapi, Krisztina Schrantz, Eszter Arany and Zsuzsanna Kozmér

5.1 Fundamental Principles of Vacuum UV Processes . . . . . . . . . . . . . . . . . . . 195

5.1.1 VUV radiation sources for water treatment $\ldots \ldots \ldots \ldots \ldots \ldots \ldots \ldots \ldots$

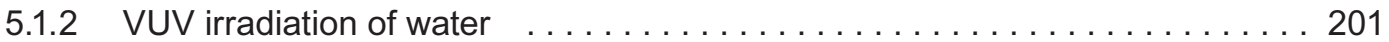

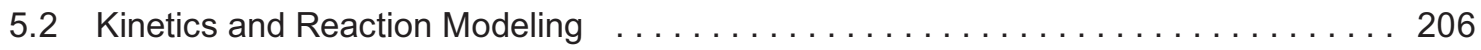

5.2.1 Reactions and role of primary and secondary formed reactive species . . . 206

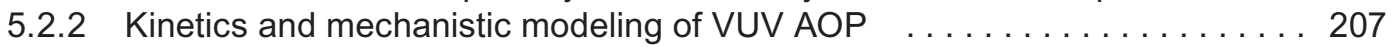

5.3 Vacuum UV Radiation for Water Remediation $\ldots \ldots \ldots \ldots \ldots \ldots \ldots \ldots$ 


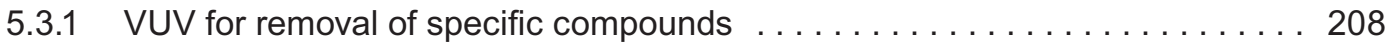

5.3.2 VUV in combination with other treatment technologies $\ldots \ldots \ldots \ldots \ldots 213$

5.4 Water Quality Impact on Vacuum UV Process Performance

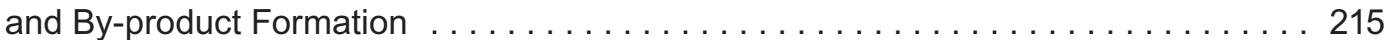

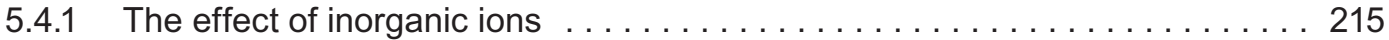

5.4.2 The effect of dissolved natural organic matter (NOM) $\ldots \ldots \ldots \ldots \ldots$

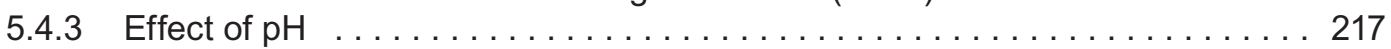

5.4.4 By-product formation during the VUV process and their removal

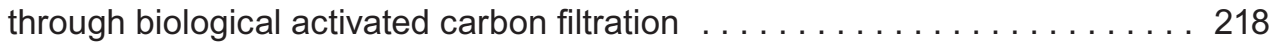

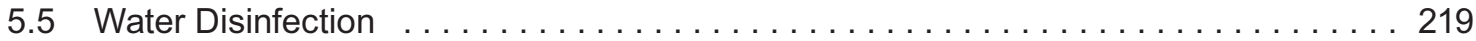

5.6 Reactor/Equipment Design and Economic Considerations . . . . . . . . . . . . . . . 220

5.6.1 Actinometry for VUV photon flow measurements . . . . . . . . . . . . 220

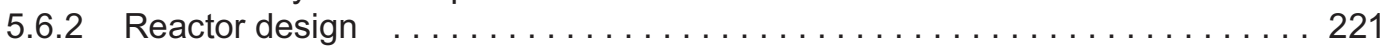

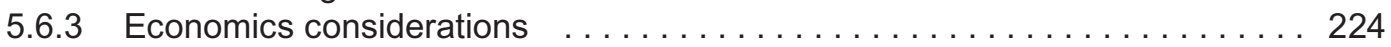

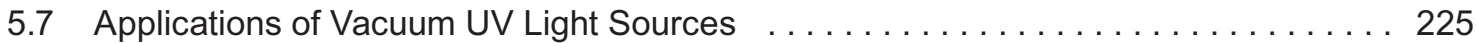

5.7.1 Applications in instrumental chemical analysis . . . . . . . . . . . . 225

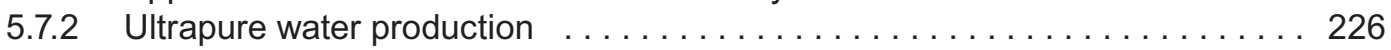

5.8 Vacuum UV AOP - General Conclusions . . . . . . . . . . . . . . . . . . . . 229

5.9 Acknowledgements . . . . . . . . . . . . . . . . . . . . . . . . . . . . . . 229

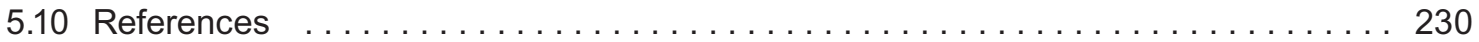

\section{Chapter 6}

Gamma-ray and electron beam-based AOPs

L. Wojnárovits, E. Takács and L. Szabó

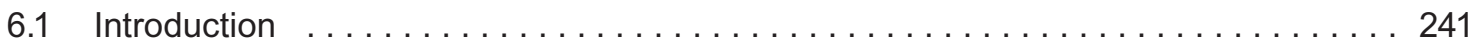

6.2 Radiolysis as a Universal Tool to Investigate Radical Reactions and as a Process for Large Scale Industrial Technology . . . . . . . . . . . . . . . . . . . . . . . 242

6.2.1 Techniques in radiation chemistry for establishing reaction mechanisms 242

6.2 .2 Sources of ionizing radiation in water treatment . . . . . . . . . . . . . 244

6.2.3 G-value, dosimetric quantities, penetration depth . . . . . . . . . . 245

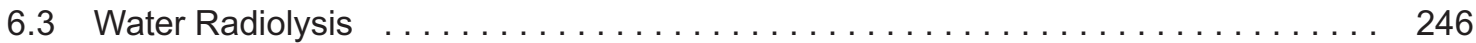

6.3.1 Process fundamentals, yields and reactions of reactive intermediates . . . 246

6.3.2 Reactions of primary species with common inorganic ions . . . . . . . . 253

6.3.3 Kinetics and modeling of ionizing radiation-induced processes . . . . . . 256

6.3.4 Toxicity of ionizing radiation-treated water . . . . . . . . . . . . . . 258

6.4 Research Studies on Water Radiolysis-Mediated Degradation of Organic Pollutants 259

6.4.1 Aromatic compounds ......................... 259

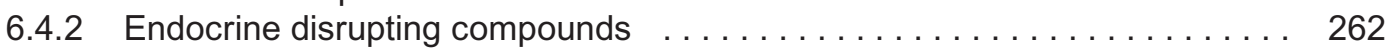

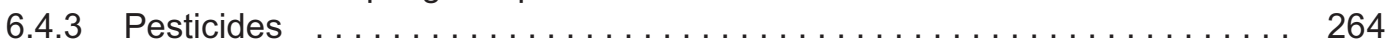

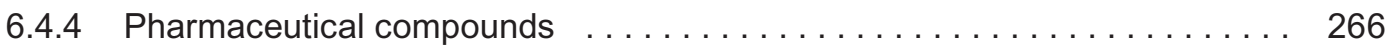

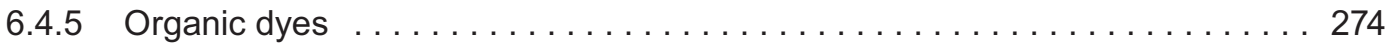

6.4.6 Naphthalene sulfonic acid derivatives . . . . . . . . . . . . . . . . 275

6.5 Ionizing Radiation for Water Treatment: Pilot- and Industrial Scale Applications . . . 276

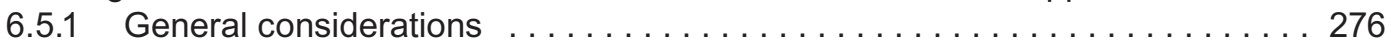

6.5 .2 lonizing radiation reactors for water treatment $\ldots \ldots \ldots \ldots \ldots \ldots \ldots$ 
6.5.3 lonizing radiation for water treatment: pilot studies $\ldots \ldots \ldots \ldots \ldots \ldots 279$

6.5.4 Industrial scale installations using radiation-based AOP $\ldots \ldots \ldots \ldots \ldots 280$

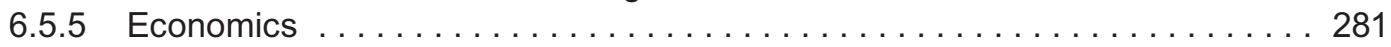

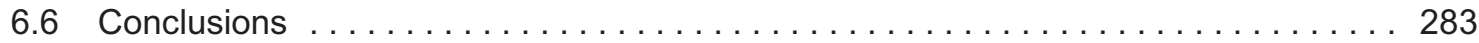

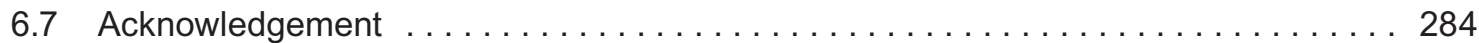

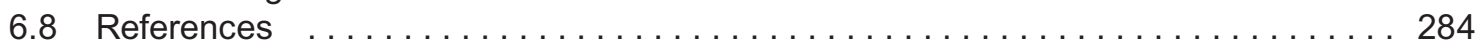

\section{Chapter 7}

Fenton, photo-Fenton and Fenton-like processes . . . . . . . . . . . 297

Christopher J. Miller, Susan Wadley, and T. David Waite

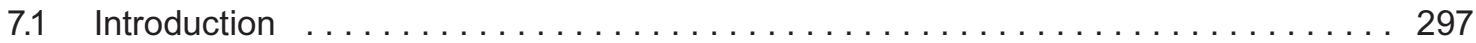

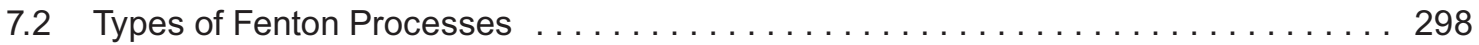

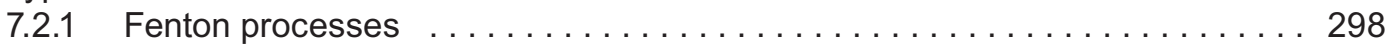

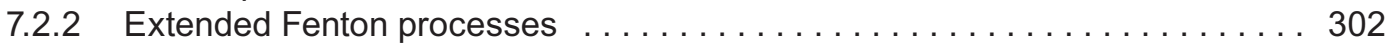

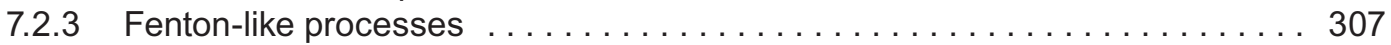

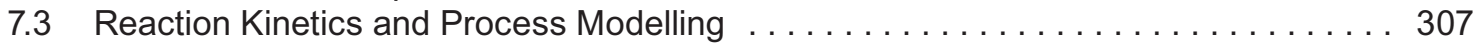

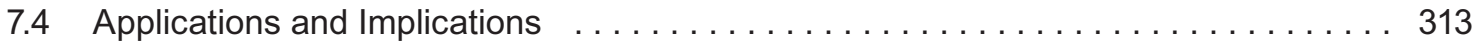

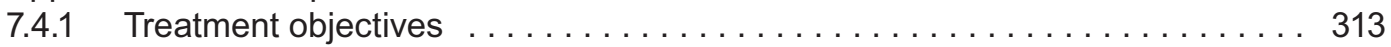

7.4.2 Types of compounds suited to treatment $\ldots \ldots \ldots \ldots \ldots \ldots \ldots \ldots \ldots \ldots \ldots$

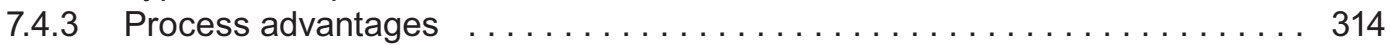

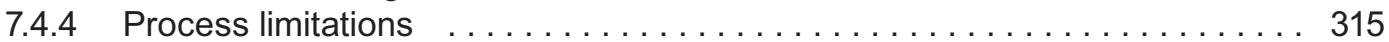

7.4.5 Laboratory and pilot plant scale studies $\ldots \ldots \ldots \ldots \ldots \ldots \ldots \ldots \ldots \ldots$

7.4.6 Commercial applications ............................ 319

7.4.7 Equipment design and economic considerations $\ldots \ldots \ldots \ldots \ldots \ldots \ldots 320$

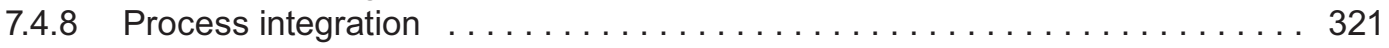

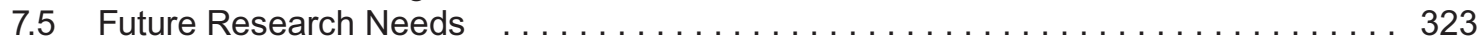

7.6 References ...................................... 323

\section{Chapter 8}

Photocatalysis as an effective advanced oxidation process . . . . . . . 333

Suresh C. Pillai, Niall B. McGuinness, Ciara Byrne, Changseok Han, Jacob Lalley, Mallikarjuna Nadagouda, Polycarpos Falaras, Athanassios G. Kontos, Miguel A. Gracia-Pinilla, Kevin O'Shea, Ramalinga V. Mangalaraja, Christophoros Christophoridis, Theodoros Triantis, Anastasia Hiskia, and Dionysios D. Dionysiou

8.1 Introduction ................................ 333

8.2 Process Principles Including the Most Recent Scientific Findings and Interpretation 334

8.2.1 Nanotubular titania-based materials for photocatalytic water and air

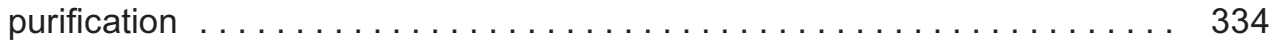

8.2.2 Magnetically separable photocatalysts $\ldots \ldots \ldots \ldots \ldots \ldots \ldots \ldots \ldots . \ldots \ldots 33$

8.2 .3 Improving the photocatalytic activity $\ldots \ldots \ldots \ldots \ldots \ldots \ldots \ldots \ldots \ldots$

8.3 Classes of Compounds Suitable to Treatment and Examples of Reaction Mechanisms ................................... 345

8.4 Kinetic Aspects, Reaction Modelling, Quantitative Structure-Activity Relationship (QSAR) 
8.5 Water Quality Impact on Process Preformance, Practical Considerations on Process

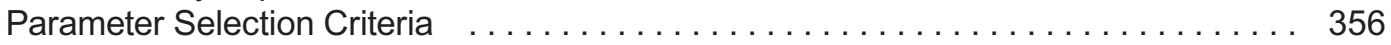

8.6 Process Limitations and Byproduct Formation; Strategies to Mitigate the Adverse Effects on the Treated Water Quality . . . . . . . . . . . . . . . . . . . . 358

8.7 Reactor/Equipment Design and Economic Considerations, Figures-of-Merit . . . . . 362

8.8 Case Studies Relevant to Process Implementation to Water Treatment . . . . . . . . 8.8.1 Contaminated groundwater with 1,4-dioxane and volatile organic solvents, Sarasota, Florida, USA (2013) . . . . . . . . . . . . . . . . . . .

8.8.2 1,4-Dioxane and VOCs destruction in drinking water,

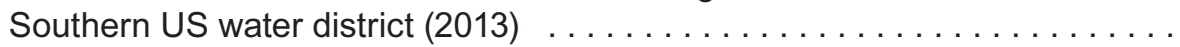

8.8.3 Removal of chromium $\left(\mathrm{Cr}^{6+}\right)$ in groundwater, Superfund site

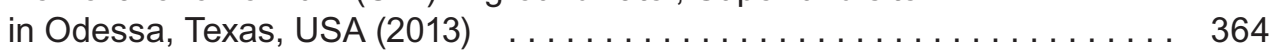

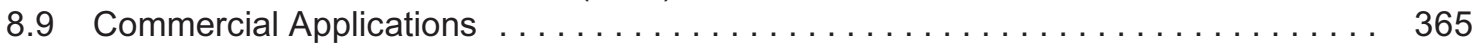

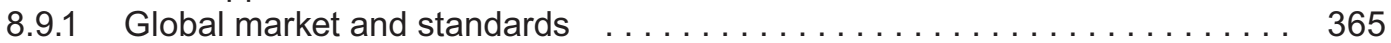

8.9.2 Drinking water regulations driving the process implementation . . . . . 365

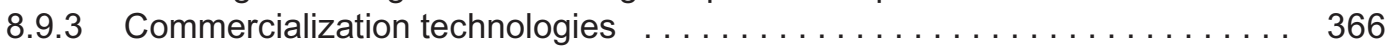

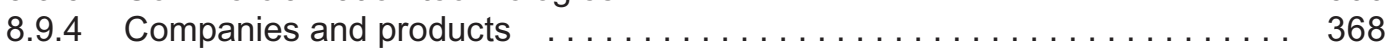

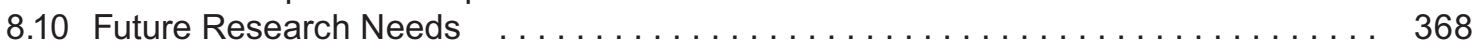

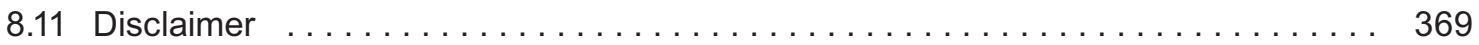

8.12 Acknowledgements ... . . . . . . . . . . . . . . . . . . . . . . . . 370

8.13 References . . . . . . . . . . . . . . . . . . . . . . . . . . . 370

\section{Chapter 9}

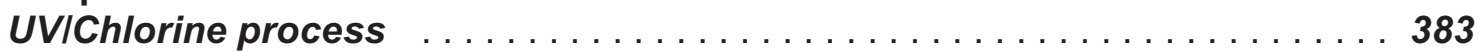

Joseph De Laat and Mihaela Stefan

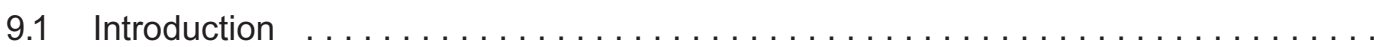

9.2 Photodecomposition of Free Chlorine by UV Light $\ldots \ldots \ldots \ldots \ldots \ldots \ldots \ldots$

9.2.1 Distribution of free chlorine species $\ldots \ldots \ldots \ldots \ldots \ldots \ldots \ldots \ldots$

9.2.2 Absorption spectra of free chlorine species in water $\ldots \ldots \ldots \ldots \ldots$

9.2.3 Radical species, quantum yields and degradation mechanisms

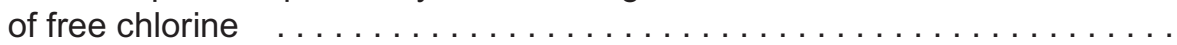

9.3 Reactivity and Fate of Chlorine Radicals $\ldots \ldots \ldots \ldots \ldots \ldots \ldots \ldots \ldots \ldots \ldots \ldots$

9.3.1 Equilibria involving the $\mathrm{Cl}^{\circ}, \mathrm{Cl}_{2}{ }^{-}{ }^{-}$and ${ }^{\circ} \mathrm{OH}$ species $\ldots \ldots \ldots \ldots \ldots \ldots . \ldots 396$

9.3.2 Termination reactions of ${ }^{\circ} \mathrm{OH}, \mathrm{Cl}^{\cdot}$ and $\mathrm{Cl}_{2}{ }^{-}{ }^{-}$in water $\ldots \ldots \ldots \ldots \ldots . .397$

9.3.3 Reactivity of $\mathrm{Cl}^{\cdot}$ and $\mathrm{Cl}_{2}{ }^{-}$towards organic and inorganic compounds . . . 393

$9.4 \quad \mathrm{UV} / \mathrm{Cl}_{2}$ Process for Contaminant Removal from Water $\ldots \ldots \ldots \ldots \ldots \ldots \ldots . \ldots 4$

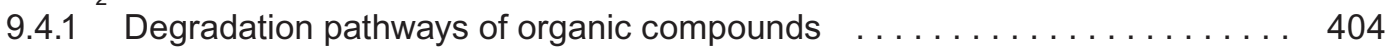

9.4.2 Kinetic modeling of $\mathrm{UV} / \mathrm{Cl}_{2} \mathrm{AOP} \ldots \ldots \ldots \ldots \ldots \ldots \ldots \ldots \ldots \ldots$

9.4.3 The impact of selected parameters on $\mathrm{UV} / \mathrm{Cl}_{2}$ process performance $\ldots \ldots 408$

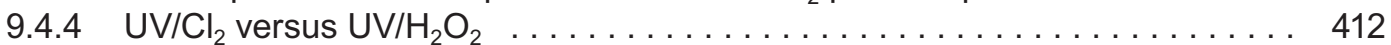

9.4.5 Byproduct formation in the UV/Cl ${ }_{2} \mathrm{AOP} \ldots \ldots \ldots \ldots \ldots \ldots \ldots \ldots . \ldots \ldots$

9.5 Research Needs . . . . . . . . . . . . . . . . . . . . . . . . . . . . . . 423

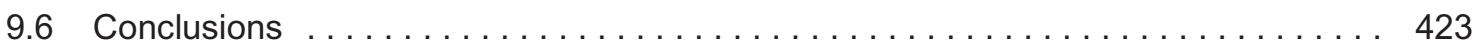

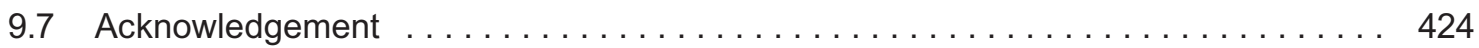

9.8 References ...................................... 424 


\section{Chapter 10}

\section{Sulfate radical ion - based AOPs}

Nathalie Karpel Vel Leitner

10.1 Introduction ... . . . . . . . . . . . . . . . . . . . . . . . . . . . . . . 429

10.2 Methods for Sulfate Radical Generation . . . . . . . . . . . . . . . . . . . . 429

10.2.1 Mild-thermal and base activation of persulfate $\ldots \ldots \ldots \ldots \ldots \ldots \ldots$

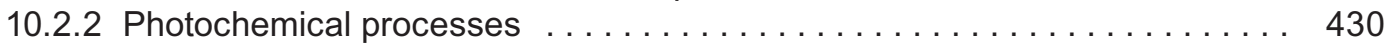

10.2.3 Transition metal-activated decomposition of persulfate salts . . . . . . 431

10.2.4 Miscellaneous processes . . . . . . . . . . . . . . . . . . . 432

10.3 Properties and Stability of Sulfate Radical in Pure Water . . . . . . . . . . . . . . . . 434

10.3.1 Oxidation-reduction potential $\ldots \ldots \ldots \ldots \ldots \ldots \ldots \ldots \ldots \ldots \ldots \ldots \ldots$

10.3.2 $\mathrm{pH}$ dependence . . . . . . . . . . . . . . . . . . . . . 435

10.4 Reaction Mechanisms with Organic Molecules in Pure Water . . . . . . . . . . . 436

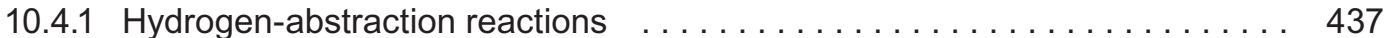

10.4.2 Electron transfer reactions $\ldots \ldots \ldots \ldots \ldots \ldots \ldots \ldots \ldots \ldots \ldots \ldots \ldots \ldots$

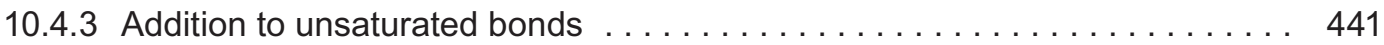

10.5 Sulfate Radical-Based Treatment of Water Micropollutants . . . . . . . . . . . . . 442

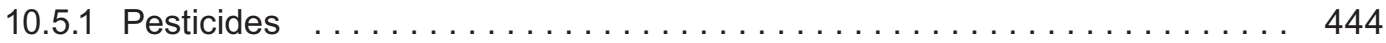

10.5.2 Pharmaceuticals ... . . . . . . . . . . . . . . . . . . . . . . . 444

10.5.3 Algal toxins and taste-and-odor (T\&O) causing compounds . . . . . . . 444

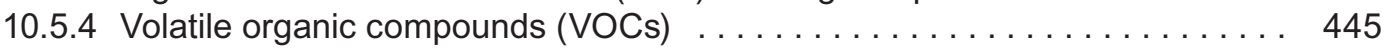

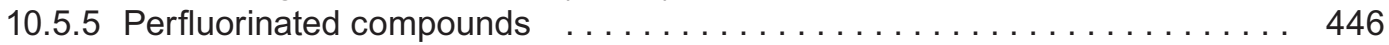

10.6 Reactions with Water Matrix Constituents in Sulfate Radical-Driven Oxidations . . 447

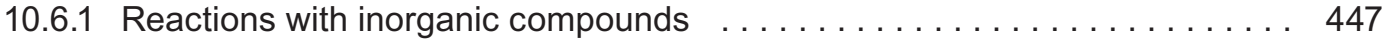

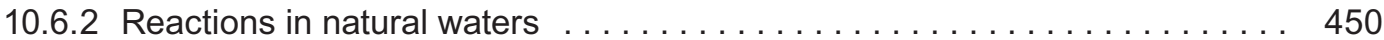

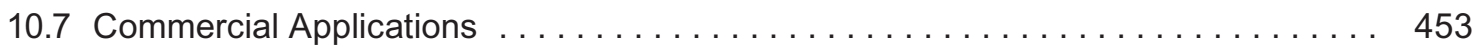

10.7.1 Total organic carbon (TOC) analyzers $\ldots \ldots \ldots \ldots \ldots \ldots \ldots \ldots \ldots \ldots$

10.7.2 In Situ Chemical Oxidation (ISCO) . . . . . . . . . . . . . . . . . 453

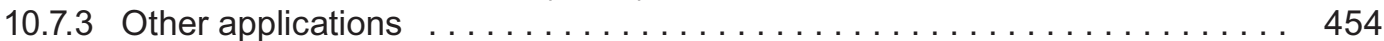

10.8 Future Research Needs . . . . . . . . . . . . . . . . . . . . . . . . . . . . . . 454

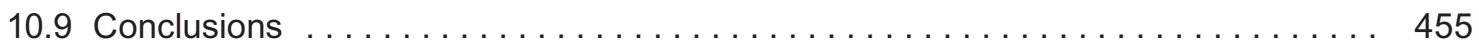

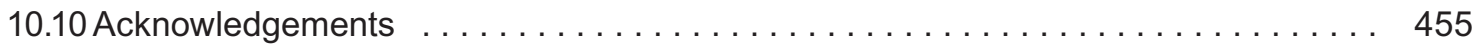

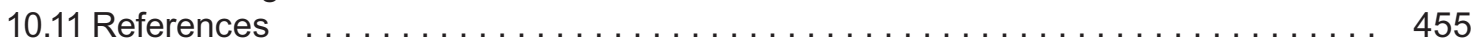

\section{Chapter 11}

Ultrasound wave-based AOPs ................... 461

O. A. Larpparisudthi, T. J. Mason and L. Paniwnyk

11.1 Introduction ..................................... 461

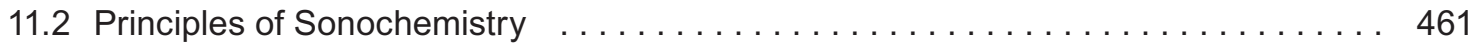

11.3 Acoustic Cavitation, the Driving Force for Sonochemistry $\ldots \ldots \ldots \ldots \ldots \ldots 463$

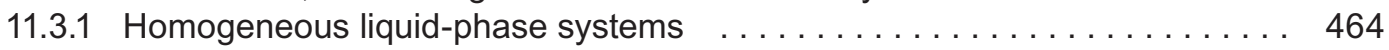

11.3.2 Heterogeneous solid surface-liquid systems $\ldots \ldots \ldots \ldots \ldots \ldots \ldots .465$

11.3.3 Heterogeneous particle-liquid systems $\ldots \ldots \ldots \ldots \ldots \ldots \ldots \ldots \ldots . \ldots \ldots 6$

11.3.4 Heterogeneous liquid-liquid systems $\ldots \ldots \ldots \ldots \ldots \ldots \ldots \ldots \ldots 466$ 
11.4 Historical Introduction on the Oxidative Properties of Ultrasound in Water . . . . 466

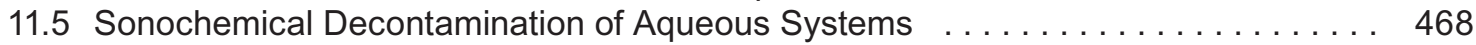

11.5.1 AOP involving ultrasound alone $\ldots \ldots \ldots \ldots \ldots \ldots \ldots \ldots \ldots \ldots \ldots \ldots$

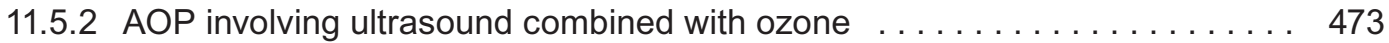

11.5.3 AOP involving ultrasound combined with ultraviolet light $\ldots \ldots \ldots \ldots$. . . 477

11.5.4 AOP involving ultrasound combined with electrochemistry . . . . . . . . 479

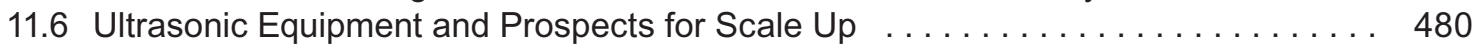

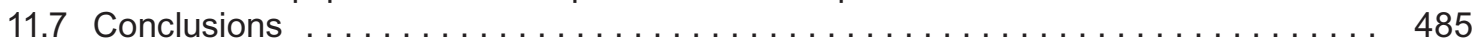

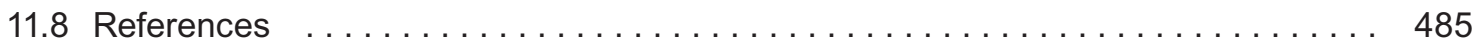

\section{Chapter 12}

Electrical discharge plasma for water treatment

Selma Mededovic Thagard and Bruce R. Locke

12.1 Introduction - Plasma Processes for Water Treatment . . . . . . . . . . . . . . . . 493

12.2 Indirect Plasma - Ozone Generation . . . . . . . . . . . . . . . . . . . . . . 495

12.3 Direct Plasma - Plasma Directly Contacts Liquid Solution . . . . . . . . . . . . . . 498

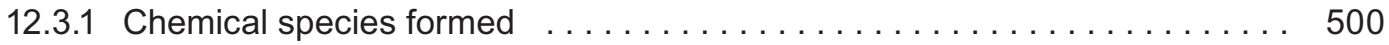

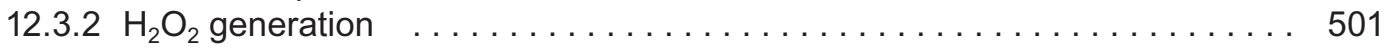

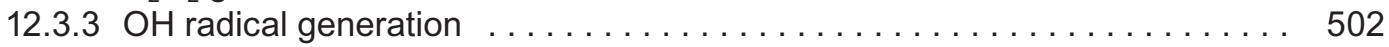

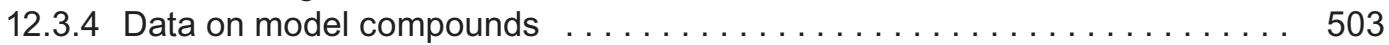

12.3.5 Thermal plasma chemistry in direct water discharges . . . . . . . . . . 515

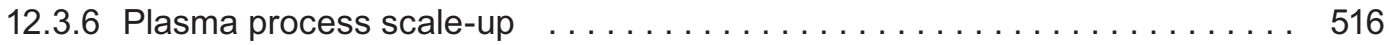

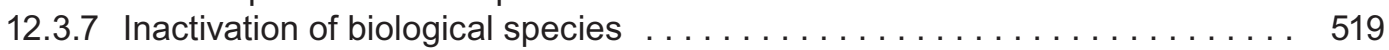

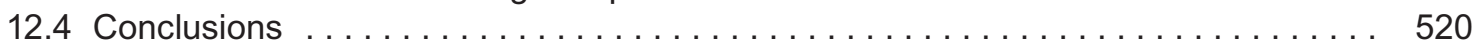

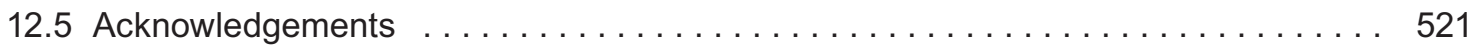

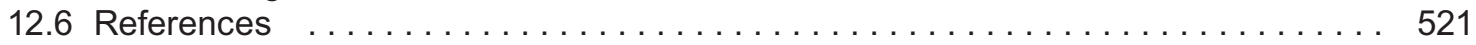

\section{Chapter 13}

The role of photochemistry in the transformation of pollutants

in surface waters . . . . . . . . . . . . . . . . . . . . . . . . . 535

Douglas E. Latch

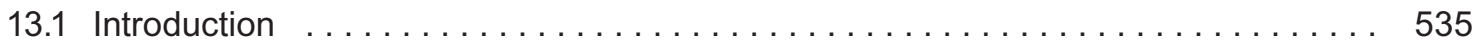

13.2 Solar Radiation at the Earth's Surface $\ldots \ldots \ldots \ldots \ldots \ldots \ldots \ldots \ldots \ldots$

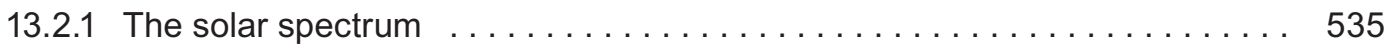

13.2.2 Diurnal, seasonal, and latitudinal variations $\ldots \ldots \ldots \ldots \ldots \ldots \ldots$

13.2.3 Light attenuation and depth dependence of photochemical reactions $\ldots \ldots 537$

13.3 Types of Photochemical Reactions in Surface Waters . . . . . . . . . . . . . . . . 537

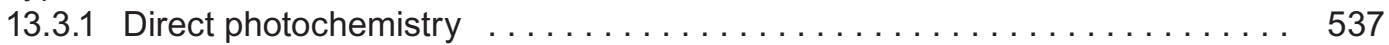

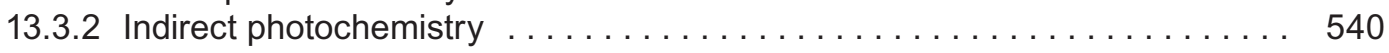

13.4 Laboratory Methods and Techniques for Studying Pollutant Photochemistry . . . . . 542

13.5 Photochemically Produced Reactive Intermediates (PPRIs) and the Role of Organic

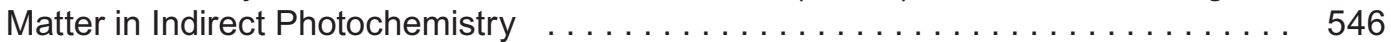

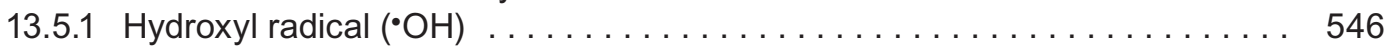

13.5.2 Excited state triplet organic matter $\left({ }^{3} \mathrm{OM}\right) \ldots \ldots \ldots \ldots \ldots \ldots$ 
13.5.3 Singlet oxygen $\left({ }^{1} \mathrm{O}_{2}\right)$

13.5.4 Hydrated electron $\left(\mathrm{e}_{\mathrm{aq}^{-}}{ }^{-}\right.$), superoxide radical anion $\left(\mathrm{O}_{2}{ }^{--}\right)$, and hydrogen peroxide

13.5.5 Carbonate radical $\left(\mathrm{CO}_{3}{ }^{-}\right) \ldots \ldots \ldots \ldots \ldots \ldots \ldots \ldots \ldots \ldots$

13.5.6 Organoperoxyl radicals $(\cdot \mathrm{O}$.

13.6 Salinity Effects on Photochemical Reactions in Natural Waters

550

13.7 Ranitidine and Cimetidine: An Illustrative Surface Water Photochemistry Examp....

13.8 Select Photochemically Active Aquatic Pollutants $\ldots \ldots \ldots \ldots \ldots \ldots \ldots$

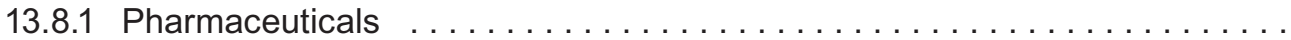

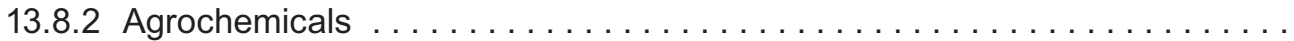

13.8.3 Other photochemically active pollutants detected in surface waters . . . . . .

13.9 Notable Examples of Aquatic Pollutants Transformed through Photochemical

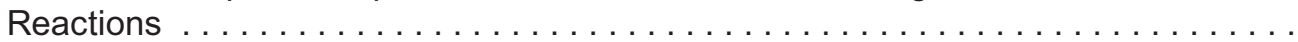

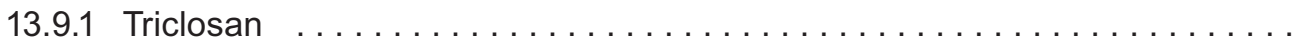

13.9.2 Steroid hormones and related EDCs $\ldots \ldots \ldots \ldots \ldots \ldots \ldots \ldots \ldots$

13.9.3 Waterborne viruses and similar model pathogens . . . . . . . . . . . . 566

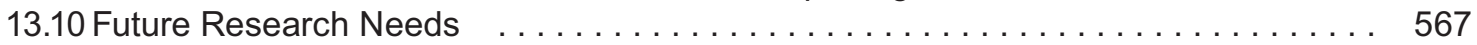

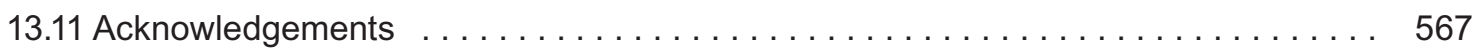

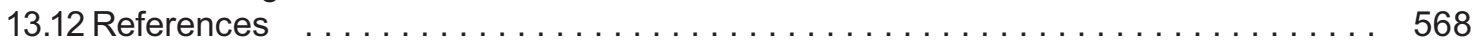

\section{Chapter 14}

Advanced treatment for potable water reuse $\ldots \ldots \ldots \ldots \ldots \ldots \ldots$

14.1 Planned Potable Water Reuse $\ldots \ldots \ldots \ldots \ldots \ldots \ldots \ldots \ldots \ldots . \ldots \ldots$

14.2 Treatment Objectives and Drivers for the Adoption of AOPs in Potable Reuse . . . 583

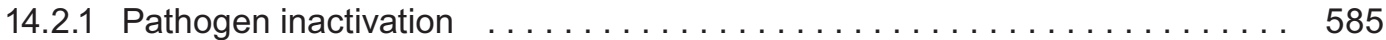

14.2.2 Trace chemical contaminants . . . . . . . . . . . . . . . . . . 586

14.3 Validation and Process Control $\ldots \ldots \ldots \ldots \ldots \ldots \ldots \ldots \ldots \ldots \ldots . \ldots \ldots \ldots$

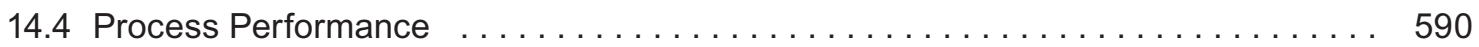

14.5 International Examples of AOP Use in Potable Reuse Projects . . . . . . . . . . 592

14.5.1 Groundwater Replenishment System, Orange County, CA, USA (2008) 592

14.5.2 Western Corridor Recycled Water Project, Queensland, Australia (2008) . . 594

14.5.3 Prairie Waters Project, Aurora, CO, USA (2010) . . . . . . . . . . . . . 597

14.5.4 Beaufort West Water Reclamation Plant (South Africa) $\ldots \ldots \ldots \ldots \ldots 598$

14.5.5 Terminal Island Water Reclamation Plant, Los Angeles, CA, USA (2016) 598

14.6 Conclusions and Future Projections $\ldots \ldots \ldots \ldots \ldots \ldots \ldots \ldots \ldots \ldots \ldots \ldots \ldots \ldots . \ldots \ldots$

14.7 References ....................................... 602

\section{Chapter 15}

Advanced treatment for drinking water production . . . . . . . . . . . . 607

Gilbert Galjaard, Bram Martijn, Erik Koreman and Holly Shorney-Darby

15.1 Introduction ... . . . . . . . . . . . . . . . . . . . . . . . . . . . . . . . . . . . . 607

$15.2 \mathrm{UV} / \mathrm{H}_{2} \mathrm{O}_{2}$ Process: Andijk Water Treatment Plant (WTP) Case Study . . . . . . . 608 
15.3 Pretreatment Strategies for AOP in Drinking Water Treatment . . . . . . . . . 611

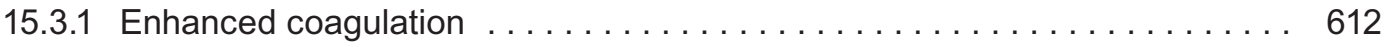

15.3 .2 Ion exchange . . . . . . . . . . . . . . . . . . . . . . 613

15.3.3 Ceramic membranes and hybrid combinations $\ldots \ldots \ldots \ldots \ldots \ldots \ldots$

15.4 The Effect of Pretreatment on MP UV/ $\mathrm{H}_{2} \mathrm{O}_{2}$ AOP $\ldots \ldots \ldots \ldots \ldots \ldots \ldots \ldots \ldots \ldots$

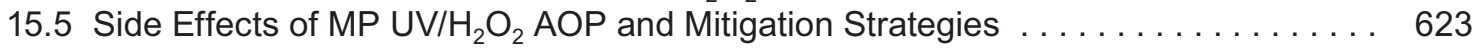

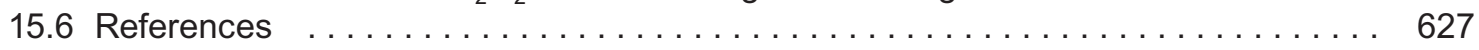

\section{Chapter 16}

AOPs for municipal and industrial wastewater treatment $\ldots \ldots \ldots \ldots 631$ Jianlong Wang and Lejin Xu

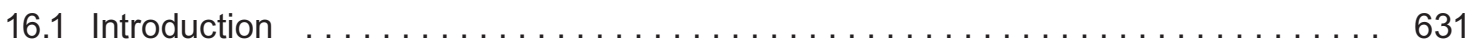

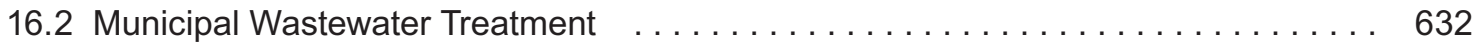

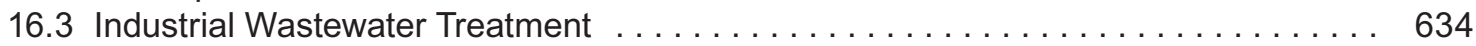

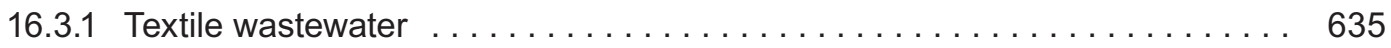

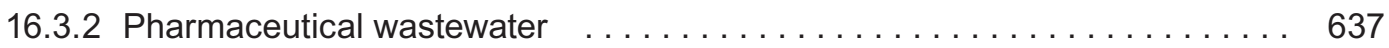

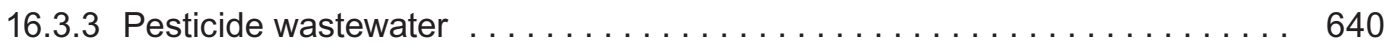

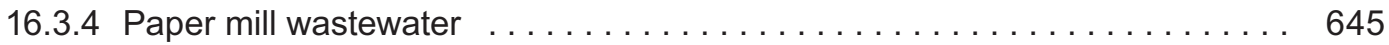

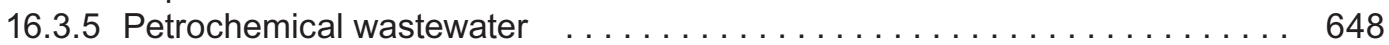

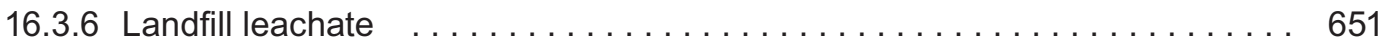

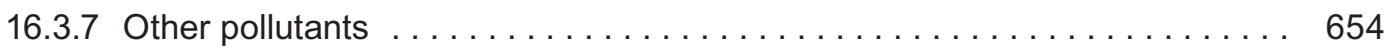

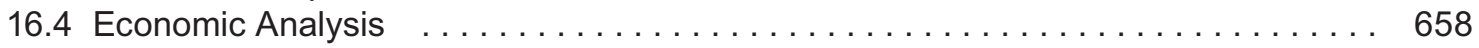

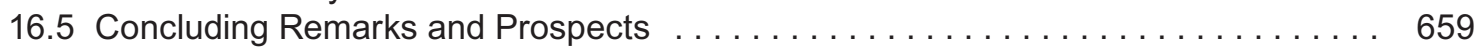

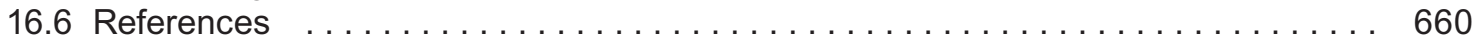

\section{Chapter 17}

Iron-based green technologies for water remediation . . . . . . . . . 667

Virender K. Sharma and Radek Zboril

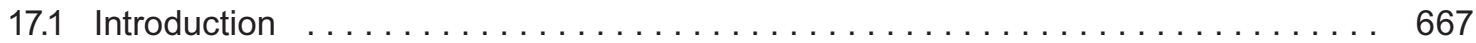

17.2 Zerovalent Iron Nanoparticles $\ldots \ldots \ldots \ldots \ldots \ldots \ldots \ldots \ldots \ldots \ldots \ldots$

17.3 Iron(III) Oxide Nanoparticles . . . . . . . . . . . . . . . . . . . . . . . . 669

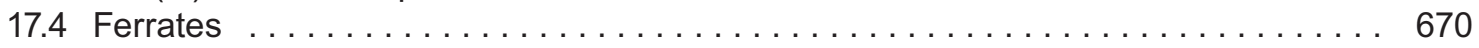

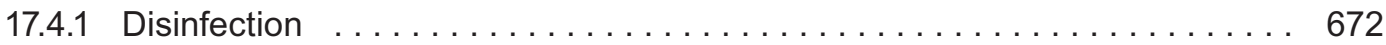

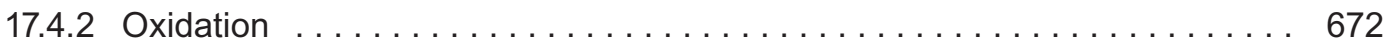

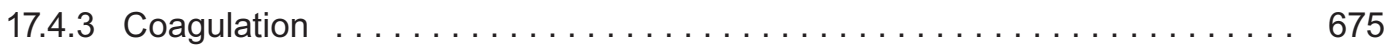

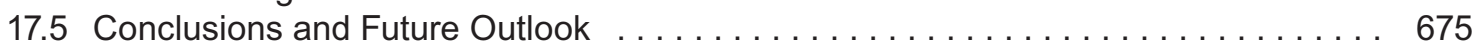

17.6 Acknowledgment . . . . . . . . . . . . . . . . . . . . . . . . . . 676

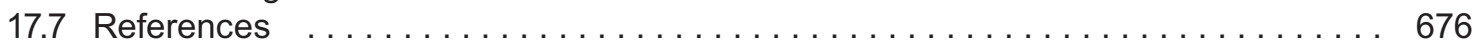

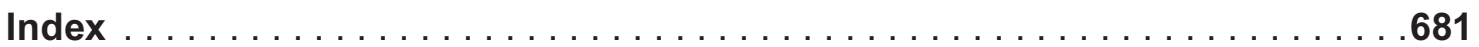

